\title{
COVID-19 and acute kidney injury - A new medical condition
}

\author{
Natália Silva ${ }^{1}$, Catarina Isabel Ribeiro ${ }^{2}$ \\ ${ }^{1}$ Nephrology Department, Trás-Os-Montes e Alto Douro Hospital Center, Vila Real - Portugal \\ ${ }^{2}$ Nephrology Department, Vila Nova de Gaia-Espinho Hospital Center, Vila Nova de Gaia - Portugal \\ Both authors contributed equally to this work
}

\section{ABSTRACT}

Novel Coronavirus disease (COVID-19) is a newly discovered contagious disease that has affected many countries around the world.

Although diffuse alveolar damage and acute respiratory failure are the main features, involvement of other organs is recognized and needs to be explored. Information on kidney disease in patients with COVID-19 is limited, with many studies showing that acute kidney injury is an uncommon disorder in COVID-19. Despite this low prevalence, it can lead to potentially significant morbidity and mortality in these patients.

Thus the authors consider the development of acute kidney injury in patients with COVID-19 an important issue, worthy of clinical attention, and they present this point in the current review.

Keywords: Coronavirus Disease 19 Infection; Severe Acute Respiratory Syndrome-CoV-2; Acute Kidney Failure

\section{INTRODUCTION}

COVID-19 is an abbreviated name for coronavirus disease 2019, suggested by the World Health Organization ${ }^{1}$. In December of 2019, this new coronavirus infection ravaged the city of Wuhan, sweeping not only through China, but also spreading to many countries around the world ${ }^{2-6}$.

COVID-19 is highly contagious and person-to-person transmission routes include droplet inhalation transmission (through coughing or sneezing), and direct contact with oral, nasal and eye mucous membranes 7,8 . The estimated incubation period is 1 to 14 days. The common clinical manifestations include fever, unproductive cough, dyspnea, myalgia, and fatigue, with accompanying leucopenia and lymphopenia ${ }^{5,7}$.

It is well documented that the lungs are the main target organ of the new coronavirus, and the incidence of acute respiratory distress syndrome in patients with COVID-19 is reported to be up to $30 \%$ of cases. However, recent reports have also revealed involvement of other organs, including kidneys, especially in severe patients ${ }^{9-11}$.

Identifying and eliminating factors predicting a negative outcome is key to improving survival from COVID-19 ${ }^{12}$; thus the authors consider the development of acute kidney injury in patients with COVID-19 an important issue worthy of clinical attention. In this manuscript they review the most recent findings of the effects of SARS-CoV-2 infection on the kidney and examined the potential factors with impact on the outcomes of these patients.

\section{METHODS}

A search was performed for clinical guidance standards, review articles, systematic reviews, meta-analyses and randomized controlled clinical trials on Medline, evidence-based medicine sites, index of Portuguese medical journals and bibliographic references of selected articles published in 2020, in Portuguese, English and Spanish, using the keywords (MeSH terms) "coronavirus infection", "severe acute respiratory syndrome-CoV-2" and "acute kidney failure".

\section{CLINICAL REVIEW}

\section{Epidemiology}

Acute kidney injury (AKI) is one of the most important complications of the 2019 novel coronavirus disease, occurring in 0.5 to $28 \%$ of cases ${ }^{1,5,10,11}$. Different studies report a highly variable prevalence of AKI in COVID-19 hospitalized patients.

Luwen Wang et al. reported the clinical characteristics of 116 hospitalized COVID-19 patients and none met the diagnostic criteria of AKI during a 1 month follow-up ${ }^{13}$. On the other hand, a recent study of 193 hospitalized COVID-19 patients with follow-up of 1.5-2 months demonstrated that kidney dysfunctions, although they might not be readily diagnosed as AKI at admission, gradually worsened during hospitalization and were later diagnosed as AKI, especially in severe patients ${ }^{11}$. 
In a prospective cohort study of 710 COVID-19 cases, more than $40 \%$ had evidence of abnormal kidney function on admission and $5.1 \%$ had AKI during their hospital stay. The incidence of AKI was significantly higher in patients with elevated baseline Scr (9.1\%) than in patients with normal baseline $\operatorname{Scr}(2.0 \%)^{12}$.

\section{Pathophysiologic mechanisms}

The genomic characterization of SARS-CoV-2 revealed a significant phylogenetic distance from previously identified coronavirus that caused human diseases, sharing only $79 \%$ and $50 \%$ identity with SARSCoV and MERS-CoV, respectively ${ }^{14,15}$.

Regardless of these differences, several studies reported that SARSCoV-2 uses the same membrane-bound angiotensin-converting enzyme 2 (ACE2) as SARS-CoV to gain access to its target cells ${ }^{16}$, although it has greater binding affinity ${ }^{17}$.

It has been described that, in the kidney, ACE2 is highly expressed in the brush border of proximal tubular cells and, to a minor extent, in podocytes, but not in glomerular endothelial and mesangial cells ${ }^{18}$.

Recent studies have reported that the human kidney is a specific target for SARS-CoV-2 infection ${ }^{19-21}$. The authors examined the viral nucleocapsid protein in situ in the kidney post-mortem and found that SARS-CoV-2 antigens accumulated in renal tubular epithelium and podocytes, suggesting that it infects the human kidney directly, inducing mitochondrial dysfunction, acute tubular necrosis, the formation of protein reabsorption vacuoles, collapsing glomerulopathy, and protein leakage in Bowman's capsule.

The greater renal tropism of SARS-CoV-2 versus SARS-CoV could be explained by the increased affinity for ACE2 ${ }^{22}$. Recently, Zhong's lab in Guangzhou successfully isolated SARS-CoV-2 from the urine sample of an infected patient, suggesting the kidney as the target of this novel coronavirus ${ }^{23}$. Varga et al. also reported that virus particles were present in renal endothelial cells, indicating viremia as a possible cause of endothelial damage in the kidney and a probable contributor to $\mathrm{AKI}^{24}$.

Some authors report that AKI could be a result of cytokine release syndrome, rather than active viral replication in the kidney ${ }^{25}$. These cytokines or mediators might exert indirect effects on renal tissue, such as hypoxia, shock, and rhabdomyolysis. What has been described so far is that a specific inhibitor of interleukin 6 (IL-6) appears to be helpful in severe cases of COVID-1926. This drug, tocilizumab, is currently being investigated in a controlled trial in China (ChiCTR2000029765) ${ }^{26}$.

Other potential mechanisms for AKI associated to SARS COV2 have been suggested: cardiorenal syndrome either due to right cardiac failure or left cardiac failure with low output, rhabdomyolysis, sepsis, alveolar damage/acute respiratory distress syndrome, and development of microemboli and microthromi in the context of hypercoagulability and endothelitis 20,27

\section{Risk factors}

A recent study investigated the differences in ACE2 gene expression between individuals in relation to race, age, sex and smoking status, in order to identify possible disparities between patients that would result in increased COVID-19 susceptibility ${ }^{28}$. Five large-scale bulk transcriptomic datasets of normal lung tissue and two single-cell transcriptomic datasets were investigated and showed that race (Asian vs. Caucasian), age (>60 vs. $<60$ ) and sex did not correlate with significant disparities in ACE2 gene expression ${ }^{28}$.

Two thirds of patients who died from COVID-19 had diabetes or cardiovascular disease, and most of these patients were treated with angiotensin-receptor blockers (ARBs) ${ }^{29}$. Some studies have demonstrated that ARBs could significantly increase ACE2 expression in the kidney and heart ${ }^{30,31}$. Could these drugs be a risk factor? An experimental study showed that the pharmacological inhibition of the angiotensin type 1 receptor with losartan attenuated severe lung injury in mice infected with SARS-CoV- $2^{32}$. Other experimental studies have supported this finding 33,34 .

\section{Clinical presentation}

Patients infected with SARS-CoV-2 are at higher risk of AKI than those infected with SARS-CoV ${ }^{22}$. Additionally, several studies revealed that proteinuria and hematuria are common features of patients on hospital admission ${ }^{11,12}$.

Zhen Li et al. have reported an elevation of plasma creatinine ( $\mathrm{SCr}$ ) and blood urea nitrogen (BUN) in 22\% and 31\% of 193 COVID-19 cases, respectively ${ }^{11}$. The duration from the onset of admission to the presence of the $\mathrm{SCr}$ increase was in the range of 0 to 20 days (median 5 days). The prevalence of proteinuria was $60 \%$ and hematuria $48 \%{ }^{11}$.

Cheng et al. recently reported that of 710 COVID-19 cases, $44 \%$ had proteinuria and hematuria and $26 \%$ had hematuria on admission $^{12}$. Serum creatinine and BUN were elevated in $14 \%$ and $13 \%$, respectively. During hospitalization, peak serum creatinine was 5.3 $\pm 3 \mathrm{mg} / \mathrm{dL}^{12}$.

Although classical assessment of AKI is still based on serum creatinine and urine output, they represent only indicators of established kidney damage. Novel biomarkers of acute tubular damage/stress such as TIMP-2 (tissue inhibitor of metalloproteinase 2) and IGFBP7 (insulin-like growth factor binding protein 7) identified using the Nephrocheck Test can improve physicians' ability to predict the risk of impending AKI in critically ill patients ${ }^{35}$.

According to existing data, the proportion of COVID-19 patients who needed renal replacement therapy ranged from 1.5\% to $17 \%$, mainly critically ill patients ${ }^{1,36}$.

\section{Treatment}

There is currently no definitive treatment for COVID-19, and the prescribed treatment regimens are the ones that have previously been effective in SARS-CoV and MERS-CoV. Currently prescribed drugs include oseltamivir, lopinavir/ritonavir, ribavirin, chloroquine phosphate and corticosteroid therapy. In a recent prospective study of 199 hospitalized adult patients with severe Covid-19, no benefit was observed with lopinavir-ritonavir treatment beyond standard care ${ }^{37}$. Chloroquine phosphate has been shown to have some efficacy against COVID-19-associated pneumonia in a multicenter clinical trial conducted in China ${ }^{38}$.

Recently, two double-blind placebo-controlled randomized clinical trials (RCT) also showed remdesivir's efficacy in shortening the time 
to recovery in adults hospitalized with severe Covid-1939,40. In June 2020, the European Medicines Agency's (EMA) committee responsible for human medicines (CHMP) recommended granting a conditional marketing authorization to remdesivir for the treatment of COVID-19 in adults and adolescents from 12 years of age with pneumonia who require supplemental oxygen.

The safety and efficacy of tociluzumab, a monoclonal antibody against the IL-6 receptor, is also undergoing evaluation by a multicenter randomized-controlled trial ${ }^{26}$.

By combining the information of different meta-analyses which included RCT, the World Health Organization (WHO) has made two recommendations for the use of corticosteroid therapy in COVID-19: a strong recommendation for systemic (i.e. intravenous or oral) therapy for 7 to 10 days in patients with severe and critical COVID-19, and a conditional recommendation not to use corticosteroid therapy in patients with nonsevere COVID-1941. The RECOVERY study, one of the largest RCT analyzed, showed the effects of dexamethasone $6 \mathrm{mg}$ given once daily (oral or intravenous) for up to 10 days in 6425 hospitalized patients with COVID-19. In the dexamethasone group, the incidence of death was lower than that in the usual care group among patients receiving invasive mechanical ventilation ( $29.3 \%$ vs. 41.4$)$ and among those receiving oxygen without invasive mechanical ventilation (( $23.3 \%$ vs. $26.2 \%)$, but not among those who were receiving no respiratory support at randomization $(17.8 \% \text { vs. } 14.0 \%)^{42}$

Based on these findings, CHMP approved dexamethasone as a treatment option for patients who require oxygen therapy (from supplemental oxygen to mechanical ventilation) in September 2020.

These drugs and various interventions that are applied to COVID-19 patients can have side effects on kidney function. e.g., invasive mechanical ventilation 43

The treatment of these patients with AKI includes general and supportive management and kidney replacement therapy. Supportive care, namely bed rest, nutritional and fluid support, and maintenance blood pressure and oxygenation are important measures.

Continuous renal replacement therapy (CRRT) has been successfully applied in the treatment of SARS, MERS and sepsis ${ }^{44,45}$. In patients with COVID-19 and AKI, early initiation of renal replacement therapy (RRT) and extracorporeal organ support (ECOS) seem to provide adequate organ support and prevent progression of disease severity. Ronco et al. published a recent review describing the potential role of extracorporeal therapies as a blood purification treatment for severe COVID-19 patients with a systemic inflammatory syndrome and multiorgan dysfunction 46 .

CRRT not only corrects hyperkalemia, volume overload and metabolic acidosis but can also adsorb cytokines and endotoxins ${ }^{45}$. Heart-kidney and lung-kidney crosstalk, as described above, can also contribute to AKI. Extracorporeal membrane oxygenation (ECMO) provides support to both heart and lungs and can be used in conjunction with CRRT ${ }^{47}$.

However, more research is needed to fully understand the benefits of these therapies in the novel coronavirus disease.

\section{Outcomes}

Recent studies revealed an association between elevated levels of proteinuria, $\mathrm{BUN}, \mathrm{SCr}$ and hematuria with higher probability of death ${ }^{11,12}$. Mortality risk for COVID-19 patients with AKI was 5 times higher than patients without $\mathrm{AKI}^{11}$.

Cheng et al. also reported a significantly higher mortality rate of COVID-19 patients with AKI (33\% vs $16 \%)^{12}$.

In a retrospective study of 2003 SARS-CoV outbreak, , AKI while described as a relatively uncommon feature of the disease, was also identified as a fatal complication of SARS, given that almost $92 \%$ of SARS patients with AKI died ${ }^{41}$.

The most important factors to avoid this outcome are to apply a high degree of vigilance, closely monitor kidney function, avoid nephrotoxins and employ supportive interventions that are renal protective at an early stage.

\section{CONCLUSION}

AKI is uncommon in COVID-19². However, it can lead to potentially significant morbidity and mortality ${ }^{3}$. The pathophysiologic mechanisms of acute kidney injury could be multifactorial, including direct infection with the SARS-CoV-2, immune and inflammatory response induced by viral infection, and systemic toxic reaction resulting from respiratory failure ${ }^{6}$.

In COVID-19 patients, kidney injury is an independent risk factor for poor prognosis ${ }^{9}$. Therefore, kidney function monitoring must be emphasized even in patients with mild respiratory symptoms, and particular attention must be given to altered kidney function after admission in clinical practice ${ }^{12}$. To reduce the mortality risk for severely ill COVID-19 patients worldwide, the authors recommend early supportive interventions. The data is still preliminary and there is a need for continued research into different aspects of viral injury to the kidney.

Author's statement: This paper reviews an area of knowledge in permanent update. The information presented here is up-to-date at the date of submission.

\section{Disclosure of potential conflicts of interest: none declared}

\section{References}

1. World Health Organization. Coronavirus disease 2019 (COVID-19) Situation Report-56. Data as reported by 16/03/2020. Available from: https://www.who.int

2. Wang L, Li X, Chen H, Yan S, Li D, Li Y, et al. Coronavirus disease 19 infection does not result in acute kidney injury: an analysis of 116 hospitalized patients from Wuhan, China. Am J Nephrol. 2020;51:343-348. doi: 10.1159/000507471

3. Mubarak1 M, Nasri H. COVID-19 nephropathy; an emerging condition caused by novel coronavirus infection. J Nephropathol. 2020; 9(3):e21. doi: 10.34172/jnp.2020.21

4. Wang D, Hu B, Hu C, Zhu F, Liu X, Zhang J, et al. Clinical characteristics of 138 hospitalized patients with 2019 novel coronavirus-infected pneumonia in Wuhan, China. JAMA. 2020; 323(11):1061 1069. doi:10.1001/jama.2020.1585.

5. Huang C, Wang Y, Li X, Ren L, Zhao J, Hu Y, et al. Clinical features of patients infected with 2019 novel coronavirus in Wuhan, China. Lancet. 2020 Feb;395(10223):497-506. doi: 10.1016/S01406736(20)30183-5

6. Zhang $F$, Liang $Y$. The potential risk of kidney vulnerable to novel coronavirus 2019 infection. Am J Physiol Renal Physiol. 2020 May 1;318(5):F1136-F1137. doi: 10.1152/ajprenal.00085.2020. 
7. Li Q, Guan X, Wu P, Wang X, Zhou L, Tong Y, et al. Early transmission dynamics in Wuhan, China, of novel coronavirus-infected pneumonia. N Engl J Med. 2020; 382:1199-1207. doi: 10.1056/NEJMoa2001316.

8. Perico L, Benigni A, Remuzzi G. Should COVID-19 concern nephrologists? why and to what extent? The emerging impasse of angiotensin blockade. Nephron. 2020; 144:213-221. doi: $10.1159 / 000507305$.

9. Xianghong $\mathrm{Y}$, Renhua S, Dechang C. Diagnosis and treatment of new coronavirus pneumonia: acute kidney injury can not be ignored. Chin Med J, 2020, 100: Online Prepublish. doi: 10.3760/ cma.j.cn112137-2020229-00520.

10. Guan WJ, Ni ZY, Hu Y, Liang WH, Ou CQ, He JX, et al. Clinical characteristics of coronavirus disease 2019 in China. N Engl J Med. doi: 10.1056/NEJMo a2002 032.

11. Li Z, Wu M, Yao J, Guo J, Liao X, Song S, et al. Caution on kidney dysfunctions of COVID-19 patients. The Lancet. February 2020. doi: 10.1101/2020.02.08.20021212.

12. Cheng Y, Luo R, Wang K, Zhang M, Wang Z, Dong L, et al. Kidney disease is associated with inhospital death of patients with COVID-19. Kidney International 2020. doi: 10.1016/j. kint.2020.03.005.

13. Luwen W, Xun L, Hui C, Shaonan Y, Dong L, Yan L, Zuojiong G. Coronavirus Disease 19 infection does not result in acute kidney injury: an analysis of 116 hospitalized patients from Wuhan China. Am J Nephrol 2020:51:343-348, doi: 10.1101/2020.02.19.20025288.

14. Lu R, Zhao X, Li J, Niu P, Yang B, Wu H, et al. Genomic characterisation and epidemiology of 2019 novel coronavirus: implications for virus origins and receptor binding. Lancet. 2020 Feb;395(10224):565-574. doi: 10.1016/S0140-6736(20)30251-8.

15. Wu A, Peng Y, Huang B, Ding X, Wang X, Niu P, et al. Genome composition and divergence of the novel coronavirus (2019-nCoV) originating in China. Cell Host Microbe. 2020 Feb;27(3):325. doi: 10.1016/j.chom.2020.02.001.

16. Hoffmann M, Kleine-Weber H, Schroeder S, Krüger N, Herrler T, Erichsen S, et al. SARSCoV-2 cell entry depends on ACE 2 and TMPRSS2 and is blocked by a clinically proven protease inhibitor. Cell. 2020 Mar. doi: DOI: 10.1016/j.cell.2020.02.052.

17. Wrapp D, Wang N, Corbett KS, Goldsmith JA, Hsieh CL, Abiona O, et al. Cryo-EM structure of the 2019-nCoV spike in the prefusion conformation. Science. 2020 Feb;367(6483):1260-1263. doi: 10.1126/science.abb2507

18. Ye M, Wysocki J, William J, Soler MJ, Cokic I, Batlle D. Glomerular localization and expression of angiotensin-converting enzyme 2 and angiotensin-converting enzyme: implications for albuminuria in diabetes. J Am Soc Nephrol. 2006 Nov;17(11):3067-3075. Doi: DOI: 10.1681/ASN.2006050423.

19. Diao B, Feng Z, Wang C, Wang H, Liu L, Wang C, et al. Human kidney is a target for novel severe acute respiratory syndrome coronavirus 2 (SARS-CoV-2) infection. medRxiv. Mar, 2020. doi: 10.1101/2020.03.04.20031120.

20. Su H, Yang M, Wan C et al. Renal histopathological analysis of 26 postmortem findings of patients with COVID-19 in China. Kidney Int. 2020;98(1):219-227.

21. Larsen CP, Bourne TD, Wilson JD, Saqqa O, Sharshir MA. Collapsing glomerulopathy in a patient with coronavirus disease 2019 (COVID-19). Kidney Int Rep 2020; published online April 9. DOI:10.1016/j.ekir.2020.04.002.

22. Wan Y, Shang J, Graham R, Baric RS, Li F. Receptor recognition by the novel from Wuhan: an analysis based on decade-long structural studies of SARS . J Virol. 2020 Jan;94(7). doi: 10.1128/ JVI.00127-20

23. The team of Zhong Nanshan responded that the isolation of SARS-CoV-2 from urine remind us to pay more attention to the cleaning of individuals and families. Guangzhou Daily. Published Febr 22, 2020. doi: 10.1016/j.kint.2020.03.001

24. Varga Z, Flammer AJ, Steiger $P$, et al. Endothelial cell infection and endotheliitis in COVID-19. Lancet 2020;395:1417-1418.

25. Tisoncik JR, Korth MJ, Simmons CP, Farrar J, Martin TR, Katze MG. Into the eye of the cytokine storm. Microbiol Mol Biol Rev. 2012 Mar;76(1):16. doi: 10.1128/MMBR.05015-11.

26. Liu R, Miller J. China approves use of Roche drug in battle against coronavirus complications. Reuters. 2020 Mar. Available from: https://www.reuters.com/

27. Zhang $\mathrm{Y}$, Xiao M, Zhang S, et al. Coagulopathy and antiphospholipid antibodies in patients with Covid-19. N Engl J Med 2020;382:e38.

28. Cai G. Bulk and single-cell transcriptomics identify tobacco-sse disparity in lung gene expression of ACE2, the receptor of 2019- nCov. Preprints. 2020. doi: 10.20944/preprints202002.0051.v2. doi: 10.20944/preprints202002.0051.v2
29. Remuzzi A, Remuzzi G. COVID-19 and Italy: what next? Lancet. 2020 Mar. doi: 10.1016/S0140$6736(20) 30627-9$.

30. Jessup JA, Gallagher PE, Averill DB, Brosnihan KB, Tallant EA, Chappell MC, et al. Effect of angiotensin II blockade on a new congenic model of hypertension derived from transgenic Ren-2 rats. Am J Physiol Heart Circ Physiol. 2006 Nov;291(5):H2166-72. doi: 10.1152/ajpheart.00061.2006.

31. Ferrario CM, Jessup J, Chappell MC, Averill DB, Brosnihan KB, Tallant EA, et al. Effect of angiotensin-converting enzyme inhibition and angiotensin II receptor blockers on cardiac angiotensinconverting enzyme 2. Circulation. 2005 May;111(20):2605-2610. doi: 10.1161/CIRCULATIONAHA.104.510461.

32. Kuba K, Imai Y, Rao S, Gao H, Guo F, Guan B, et al. A crucial role of angiotensin converting enzyme 2 (ACE2) in SARS coronavirus-induced lung injury. Nat Med. 2005 Aug;11(8):875-879. doi: 10.1038/ nm1267.

33. Gu H, Xie Z, Li T, Zhang S, Lai C, Zhu P, et al. Angiotensin-converting enzyme 2 inhibits lung injury induced by respiratory syncytial virus. Sci Rep. 2016 Jan;6:19840. doi: 10.1038/srep19840.

34. Towler P, Staker B, Prasad SG, Menon S, Tang J, Parsons T, et al. ACE2 X-ray structures reveal a large hinge-bending motion important for inhibitor binding and catalysis. J Biol Chem. 2004 Apr;279(17):17996-8007. doi: 10.1074/jbc.M311191200.

35. Zhang $\mathrm{Y}$, Xiao $\mathrm{M}$, Zhang $\mathrm{S}$, et al. Coagulopathy and antiphospholipid antibodies in patients with Covid-19. N Engl J Med 2020;382:e38.

36. Yang $X, Y u Y, X u J$, et al. Clinical course and outcomes of critically ill patients with SARS-CoV-2 pneumonia in Wuhan, China: a single-centered, retrospective, observational study [J]. Lancet Respir Med, 2020. doi: 10.1016/\$2213-2600(20)30079-5.

37. Expert Team of Chinese Society of Nephrology. Expert consensus on diagnosis and treatment of 2019 novel coronavirus (2019 - nCoV) infection with acute kidney injury. Chin J Nephrol. 2020; $97,824-828$.

38. Gao J, Tian Z, Yang X. Breakthrough: chloroquine phosphate has shown apparent efficacy in treatment of COVID-19 associated pneumonia in clinical studies. Biosci Trends. 2020;14:72-73. doi: 10.5582/bst.2020.01047.

39. Beigel JH, Tomashek KM, Dodd LE, Mehta AK, Zingman BS, Kalil AC, et al. Remdesivir for the treatment of Covid-19 - preliminary report. N Engl J Med. 2020;

40. Wang Y, Zhang D, Du G, Du R, Zhao J, Jin Y, et al. Remdesivir in adults with severe CoVID-19: a randomised, double-blind, placebo-controlled, multicentre trial. Lancet [Internet] 2020;395(10236):1569-1578.

41. Who Health Organization, Corticosteroids for COVID-19, Living Guidance 2 September 2020; file://C:/Users/natal/Downloads/WHO-2019-nCoV-Corticosteroids-2020.1-eng.pdf

42. The RECOVERY Collaborative Group. Dexamethasone in hospitalized patients with Covid-19preliminary report. N Engl J Med July 17, 2020. DOI: 10.1056/NEJMoa2021436

43. Van den Akker JPC, Egal M, Groeneveld ABJ. Invasive mechanical ventilation as a risk factor for acute kidney injury in the critically ill: a systematic review and meta-analysis. Crit Care 2013;17(3):R98-R. doi: 10.1186/cc12743.

44. Chu KH, Tsang WK, Tang CS, Lam MF, Lai FM, To KF, et al. Acute renal impairment in coronavirusassociated severe acute respiratory syndrome. Kidney Int. 2005 Feb;67(2):698-705. doi: 10.1111/j.1523-1755.2005.67130.x.

45. Arabi YM, Arifi AA, Balkhy HH, et al. Clinical course and outcomes of critically ill patients with Middle East respiratory syndrome coronavirus infection. Ann Intern Med. 2014;160:389-397. doi: 10.7326/M13-2486

46. Ronco C, Navalesi P, Vincent JL. Coronavirus epidemic: preparing for extracorporeal organ support in intensive care[J]. Lancet Respir Med, 2020. Doi: 10.1016/\$2213-2600(20)30060-6.

47. Ronco C, Reis T. Kidney involvement in COVID-19 and rationale for extracorporeal therapies. Nat Rev Nephrol 2020;16(6):308-310.

\section{Correspondence to:}

Natalia Silva, MD

Nephrology Department, Trás-Os-Montes e Alto Douro Hospital Center

Av. Noruega, Lordelo, 5000-508 Vila Real, Portugal

Email: nataliasofia_1@hotmail.com) 